\title{
Trends in Inequality in Cigarette Smoking Prevalence by Income According to Recent Anti-smoking Policies in Korea: Use of Three National Surveys
}

\author{
Youngs Chang ${ }^{1}$, Sanghyun Cho' ${ }^{1}$ Ikhan Kim ${ }^{1}$, Jinwook Bahk ${ }^{2}$, Young-Ho Khang ${ }^{1,3}$ \\ ${ }^{1}$ Department of Health Policy and Management, Seoul National University College of Medicine, Seoul, Korea; ${ }^{2}$ Department of Public Health, \\ Keimyung University, Daegu, Korea; ${ }^{3}$ Institute of Health Policy and Management, Seoul National University Medical Research Center, Seoul, Korea
}

Objectives: This study examined trends in inequality in cigarette smoking prevalence by income according to recent anti-smoking policies in Korea.

Methods: The data used in this study were drawn from three nationally representative surveys, the Korea National Health and Nutrition Examination Survey, the Korea Community Health Survey, and the Social Survey of Statistics Korea. We calculated the age-standardized smoking prevalence, the slope index of inequality, and the relative index of inequality by income level as a socioeconomic position indicator.

Results: Smoking prevalence among men decreased during the study period, but the downward trend became especially pronounced in 2015, when the tobacco price was substantially increased. Inequalities in cigarette smoking by income were evident in both genders over the study period in all three national surveys examined. Absolute inequality tended to decrease between 2014 and 2015 among men. Absolute and relative inequality by income decreased between 2008 and 2016 in women aged 30-59, except between 2014 and 2015.

Conclusions: The recent anti-smoking policies in Korea resulted in a downward trend in smoking prevalence among men, but not in relative inequality, throughout the study period. Absolute inequality decreased over the study period among men aged 30-59. A more aggressive tax policy is warranted to further reduce socioeconomic inequalities in smoking in young adults in Korea.

Key words: Income, Policy, Smoking, Socioeconomic factors, Korea

\section{INTRODUCTION}

Received: September 27, 2018 Accepted: October 22, 2018

Corresponding author: Young-Ho Khang, MD, PhD Department of Health Policy and Management, Seoul National University College of Medicine, 103 Daehak-ro, Jongno-gu, Seoul 03080, Korea

E-mail: yhkhang@snu.ac.kr

This is an Open Access article distributed under the terms of the Creative Commons Attribution Non-Commercial License (http://creativecommons.org/licenses/by$\mathrm{nc} / 4.0 / /$ which permits unrestricted non-commercial use, distribution, and reproduction in any medium, provided the original work is properly cited.
Cigarette smoking increases the risk of developing not only lung cancer, but also ischemic heart disease, cerebrovascular disease, lower respiratory infections, chronic obstructive pulmonary disease, and tuberculosis, and as such it poses one of the most serious threats to public health $[1,2]$. According to the Global Burden of Disease Study, smoking was the risk factor that caused the second-greatest amount of premature deaths and disabilities globally in 2016, following dietary factors $[3,4]$. 
Smoking prevalence tends to be distributed unequally across socioeconomic groups, causing socioeconomic inequalities in health outcomes. Studies in European countries have demonstrated a significant decrease in tobacco consumption among higher socioeconomic groups over time $[5,6]$. People from lower socioeconomic groups are likely to have a higher mortality rate related to smoking [7]. Kivimaki et al. [8] showed in their cohort study using the Whitehall cohort data that absolute inequalities in coronary heart disease mortality would be reduced by $43 \%$ if smoking was eliminated from all socioeconomic groups. A New Zealand study reported that increased inequalities in cancer mortality, particularly in lung cancer, were the main driver of increased socioeconomic inequalities in overall mortality [9]. A Korean study also indicated that cigarette smoking had a larger impact on absolute inequalities in all-cause and cardiovascular mortality than other factors such as high blood pressure, high blood glucose, and high body mass index [10].

Studies on the impact of tobacco control interventions on socioeconomic inequalities in smoking have mostly been conducted in Western countries. Raising tobacco prices is considered to be among the most effective and sustainable interventions [11-14]. Similar studies have been conducted in Korea. Cho et al. [15] reported that socioeconomic health inequalities in cigarette smoking increased between 1990 and 1998 among men public servants aged 30-49. Khang and Cho [16] showed that tobacco control policies between 1989 and 2003 most effectively reduced smoking prevalence among Korean men, while socioeconomic disparities in cigarette smoking continued to increase in both men and women despite the various anti-smoking measures implemented between 1995 and 2006 [17]. That study also provided evidence that the pace of increase in the magnitude of inequalities tended to slow down around 2005, when the cigarette price was raised by $20 \%$ [16].

Korea has established several anti-smoking policies since the 1990s, as listed in Table S1. In 1995, the government introduced the National Health Promotion Act to regulate smoking in public spaces [18]. In 1999, levies for the National Health Promotion Fund were imposed on cigarettes. In 2003, Korea joined the Framework Convention on Tobacco Control, led by the World Health Organization, and the government devised a more comprehensive tobacco control plan [19]. In 2004, tobacco prices were increased by Korean won (KRW) 500 (US\$ 0.5) and all public facilities were designated as smoke-free zones in 2012.
In 2015, indoor smoking was also banned in all businesses and restaurants regardless of their size, along with another round of price hikes, moving the price of a pack of cigarettes up from KRW 2500 (US\$ 2.3) to KRW 4500 (US\$ 4.0), the boldest move so far.

Only a few studies have conducted a time trend analysis of inequalities in smoking prevalence in relation to tobacco control policies in Korea. The most recent cigarette price hike in Korea is considered a highly proactive anti-smoking measure, which means that an analysis of its impacts on smoking inequalities will have international implications. Most studies analyzing inequalities in smoking over time have used a single source of data. However, analyzing inequality trends with various sources of data will improve the robustness of the study findings, considering the different data collection methods and sample sizes of each data set. This study probed three nationally recognized survey data sets to identify the impacts of the most recent anti-smoking policy on inequalities in smoking in Korea.

\section{METHODS}

\section{Data}

The data used in this study were drawn from three nationally representative surveys containing questions on health behaviors: the Korea National Health and Nutrition Examination Survey (KNHANES), the Korea Community Health Survey (KCHS), and the Social Survey of Statistics Korea (SSSK). The KNHANES, the KCHS, and the SSSK are nationally representative surveys conducted annually. These three national surveys annually provide individual weights to represent the national population. The results of the KNHANES and the KCHS between 2009 and 2015 (2016 for the KNHANES) were selected, as well as the 2008, 2010, 2012, 2014, and 2016 data from the SSSK. We integrated two years of data from the KNHANES to secure a sufficient sample size for analysis. Men and women respondents aged 30 years and older were included as study subjects. The total number of subjects were 40579,1415915 , and 148216 , respectively, for each survey, excluding any missing values in the source data. Informed consent for participation in all three surveys was granted by all subjects.

\section{Socioeconomic Position}

Income level was used as the indicator of socioeconomic position, because socioeconomic inequalities in smoking fol- 
lowing a price hike were expected to be most recognizable using income level as an indicator. Studies on education level and occupational class have been presented in previous studies, and analyses using these parameters have shown similar trends to those obtained by analyzing income [20]. Equivalized income, considering household size, was used to represent income. Income levels were categorized into tertiles to obtain stable results, considering the relatively small sample size of the KNHANES. Missing income values were imputed with estimates using the regression method, adjusting for age, gender, education level, and occupation. The number of missing values was 652 in the KNHANES and 71547 in the KCHS.

\section{Outcome Variable}

In the KNHANES and the KCHS, we defined current smokers as those who responded that they smoked every day or occasionally, among those who had smoked a total of 5 packs or 100 cigarettes in their life. In the SSSK survey, where no question assessed the total number of cigarettes consumed throughout a respondent's lifetime, current smokers were defined as those who currently smoked. Tobacco price could affect both smoking initiation and quitting, and thereby impact smoking prevalence. For this reason, we used smoking prevalence as the outcome measure. Studies examining the impact on smoking initiation and quitting are needed in the future.

\section{Statistical Analysis}

The study subjects included adults of both genders aged 30 and over. Subjects were grouped by gender and age (30-59 and 60 or older). Khang and Cho [16] found that trends in socioeconomic inequality in cigarette smoking varied by age. Age-standardized smoking prevalence was calculated, with 10-year groups based on the 2010 census data. The magnitude of inequalities was then evaluated in absolute and relative terms. The prevalence difference (PD), prevalence ratio (PR), slope index of inequality (SII), and relative index of inequality (RII) were calculated for each year and age group. SII and RII were calculated to represent the magnitude of absolute and relative inequalities, respectively, using the information on the cumulative distribution of age-group specific equivalized income [21]. SII represents the absolute difference in value between the lowest and the highest ends of socioeconomic position, while RII represents the ratio of the prevalence between the highest and lowest ends [21-23]. The link identity function of Proc GENMOD in SAS was used to calculate SII and $P D$, while the link log function was used for calculating RII and $P R$. Convergence availability was confirmed for all calculations of PD, PR, SII, and RII. Sample weights were taken into account in all calculations. Those aged 60 or over for both genders in the KNHANES data were not examined due to the insufficient number of smokers. For instance, the number of men aged 60 years and older who smoked was only 328 in the 2015 and 2016 data combined. The number of women aged 60 years and older who smoked was 59 in the 2015 and 2016 data, even when two years of data were combined. For the KNHANES data, the analysis was made after combining two sequential calendar years of data: 2009 and 2010, 2011 and 2012, 2013 and 2014, and 2015 and 2016, respectively. The years of data for the KCHS were 2009 to 2015 . The years of data for the SSSK were 2008, 2010, 2012, 2014, and 2016. The analysis was conducted in SAS version 9.4 (SAS Institute Inc., Cary, NC, USA) and R 3.4.2 (https://cran.r-project.org/bin/windows/base/old/ 3.4.2/) were used for the analysis.

\section{RESULTS}

The general characteristics of the subjects of the KNHANES, the KCHS, and the SSSK are presented in Tables S2-S4, respectively. Annual smoking prevalence by gender and age group is presented in Table S5. Men aged 30-59 had an approximately $20 \%$ higher prevalence than men aged 60 and over. The results from all three data sets suggested downward trends in cigarette consumption in both age groups of men participants, with significant dips around 2015, where tobacco prices were raised from KRW 2500 (US\$ 2.3) to KRW 4500 (US\$ 4.0) per pack. For women, the gap between two age groups was not as pronounced as was the case for men. The smoking prevalence among women in the older age group decreased when the first and last years of the survey data were compared. Among the younger group of women subjects, smoking prevalence was higher in the KNHANES data than in the other two data sets, while the same was not true among the older group of women subjects.

Table 1 demonstrates trends in age-standardized smoking prevalence by gender and age group according to income tertiles. The data from the three surveys showed a trend for smoking prevalence to decrease as the income level increased, providing evidence for inequalities in smoking by income. 


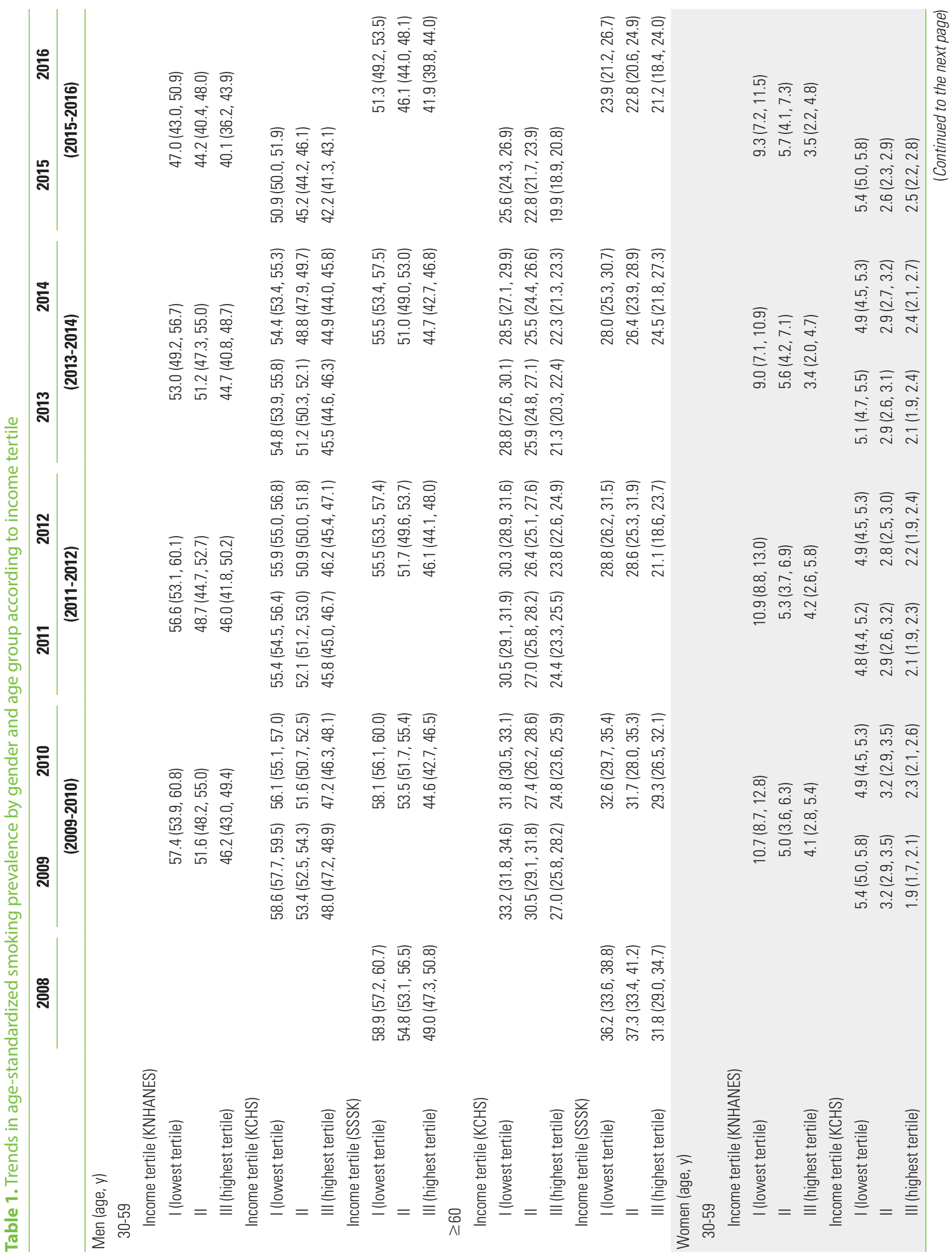




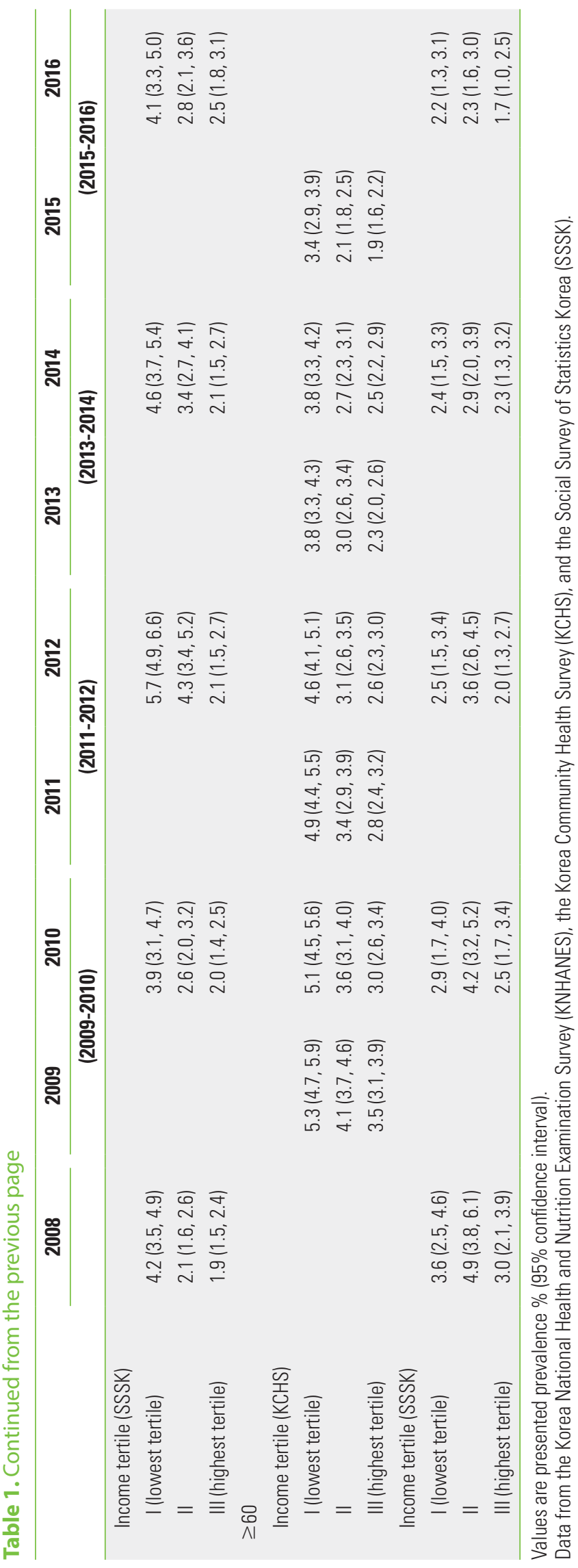

Analysis of all three survey results suggested downward trends in smoking prevalence in both low- and high-income groups among men of both age groups. Meanwhile, such a trend was seen only in the older age group among women. The KNHANES analysis of women aged 30-59 demonstrated a decrease in smoking prevalence in the highest and lowest income tertiles when comparing data from the first and last years of the survey.

Table 2 is a summary of trends in SII and RII by gender and age group according to income levels. When comparing the first and last years of survey data among men aged 30-59, SII fell from 16.6 to 10.8 in the KNHANES data, and from 15.7 to 13.0 in the KCHS data; the magnitude of inequalities decreased after 2015, when the historic price hike took place. In the SSSK data, SIl dropped from 16.2 to 14.5 between 2014 and 2016, although it was still slightly higher than the SII value of 14.3 that was recorded in 2008. In contrast, RIl tended to move in different directions in the three survey data sets. The RII dropped from 1.36 in 2009-2010 to 1.27 in 2015-2016 in the KNHANES data, while the opposite was found in the SSSK data, where it rose from 1.29 to 1.36 . Rll remained stable in the KCHS data. SIl moved downwards in the KCHS and the SSSK data among men aged 60 and over, while Rll showed upward trends.

SIl among women aged 30-59 slightly increased from 8.6 to 8.7 in the KNHANES data, decreased from 4.9 to 3.8 in the KCHS data, and decreased from 2.9 to 2.5 in the SSSK data, when comparing the first and last years of each survey. Between 2014 and 2015, the SII increased from 8.0 to 8.7 in the KNHANES, and from 3.5 to 3.8 in the KCHS. Meanwhile, the RII decreased when the first and last years of the data were compared in all three surveys, but the opposite trend was found between 2014 and 2015. During this period, the RII moved upwards from 4.39 to 4.46 in the KNHANES data and from 3.12 to 3.48 in the KCHS data, while the opposite was true in the case of the SSSK data, in which it slid from 3.23 to 2.40. Among women aged 60 and older, SII had an inverse correlation with income in both the KNHANES and the SSSK results, except for the period between 2014 and 2015. RII showed an upward trend in both surveys.

Table 3 showcases how the four inequality indicators (PD, PR, SII, RII) changed year-on-year by age group and socioeconomic status in the KNHANES, the KCHS, and the SSSK data sets. 


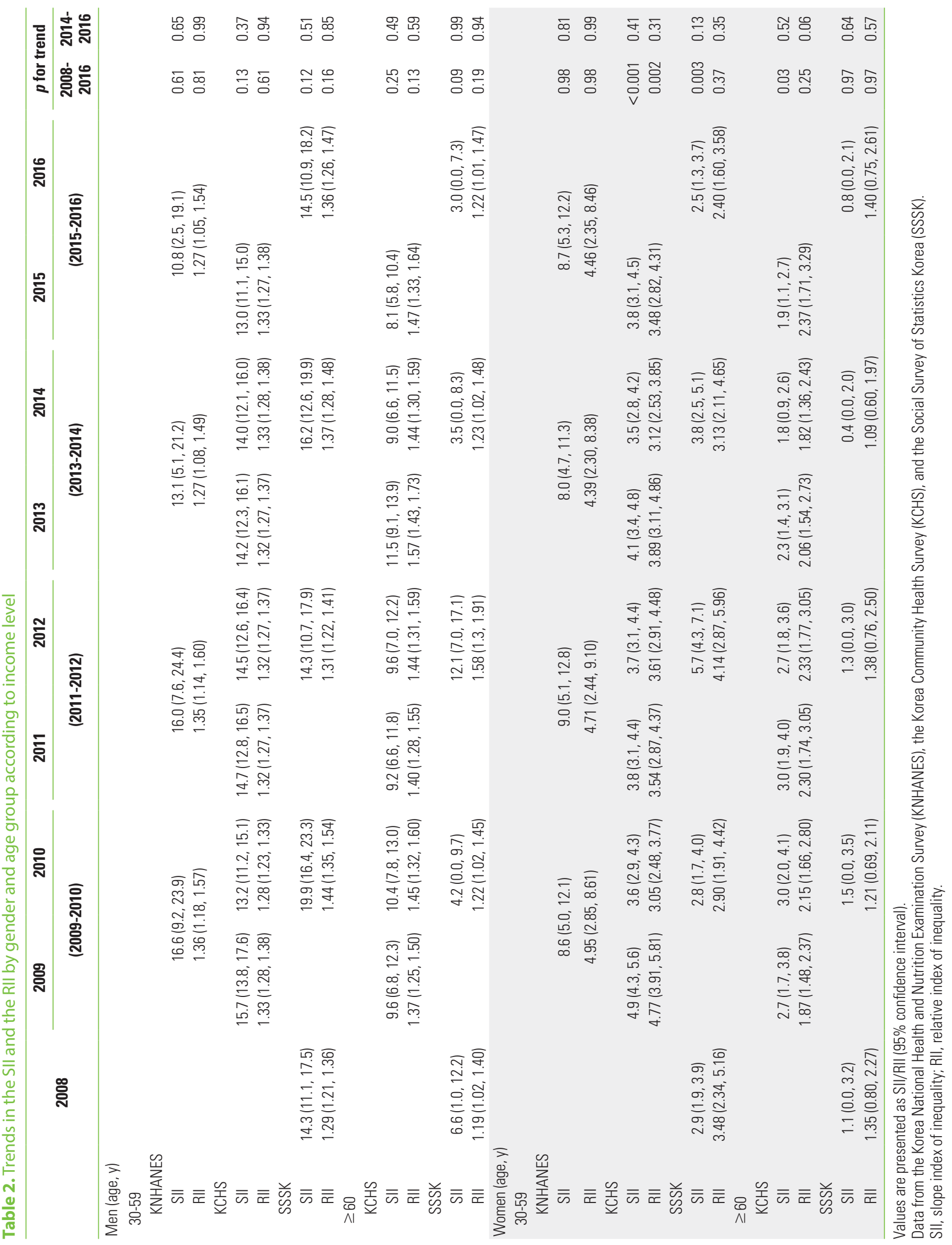




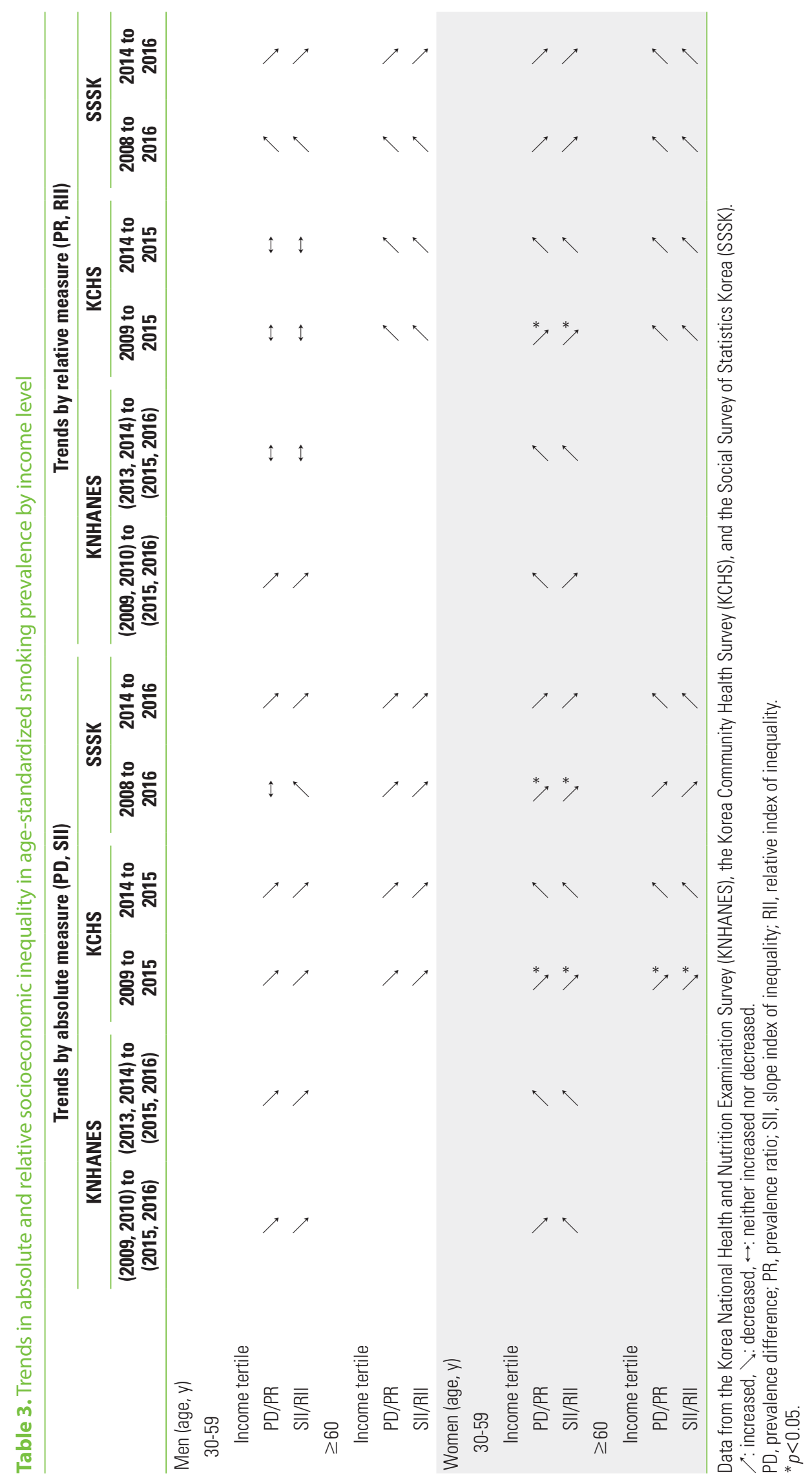




\section{DISCUSSION}

The results of this study confirmed that the absolute prevalence of smoking was higher among men than women, and among men aged 30-59 than their older counterparts aged 60 and older. This trend was seen consistently in all three surveys and in all survey years. The smoking prevalence among men also diminished over time in all three data sets. Such results are in line with those of previous studies [1,3,15-17]. Smoking prevalence among women, however, showed inconsistent trends between age groups. There was no clear trend of declining prevalence year-on-year among women between 30 and 59 years old, unlike among their older counterparts (60 years and above). When a comparison was made between 2014 and 2015, when cigarette packs became significantly more expensive, the smoking prevalence among men took a sharp dive in both age groups, while only those aged 60 and above seemed to have been impacted in the case of women smokers.

The KNHANES data showed fluctuating findings for smoking prevalence by income, due to its relatively small sample size. Inequalities in smoking were identified from the analysis of the KCHS and the SSSK data, which suggested an inverse relationship between income and smoking prevalence in all gender and age groups. Looking at detailed trends of the SII and RII figures, SIl decreased in both age groups for men and in the 60-years-and-above age group for women. Between 2014 and 2015, SIl decreased among men, but increased among women. Rll only decreased among the younger women group as calendar year increased, while it plateaued or increased in other groups. Between 2014 and 2015, the RII became higher in both genders.

Absolute inequalities in smoking among men decreased between 2014 and 2015, although the gap was not statistically meaningful, while relative inequalities remained at the same level or grew slightly. The difference between the absolute inequality (SII) and the relative inequality (RII) might have partially been due to the declining overall smoking prevalence, which limited the range of SII, but not RII. Amid the overall declining tendency of smoking prevalence among men, the movement was most pronounced in 2015. In the same year, the increasing trend of inequalities also took a slight downturn. The smoking prevalence among men might have been more directly affected by the recent anti-smoking policies than that among women because women's smoking prevalence in Korea could be influenced by other factors, including social stigma, cultural factors, and underreporting. This implies that the recent regulatory measures had an impact on men's smoking prevalence and inequalities. In terms of inequalities by income level, there was no indication of reduced inequality between 2014 and 2015 in either age group among women in absolute or in relative terms. This suggests that the recent tobacco control measure had little effect on absolute inequalities by income among Korean women.

The reason why the recent hike in cigarette prices was not especially successful in reducing smoking prevalence and inequalities, especially among women, seems to be that cigarettes are still relatively affordable in Korea. According to Organization for Economic Cooperation and Development (OECD) data, the price of a pack of cigarettes, priced at KRW 4,500 (US\$ 4.0) in Korea, was one of the lowest values among the member countries [24].

Several reasons might be considered to explain the patterns of smoking prevalence and its inequality among Korean women. Declining social stigma against young women's smoking in Korea might have resulted in an increased or stagnant smoking prevalence among young women. The stigma might have had different impacts on different income groups among women. Although fewer than $10 \%$ of women respondents responded that they were current smokers in all three surveys, it is likely that this number is an underrepresentation of the actual number. Korea's men-centered culture may prompt women smokers to avoid admitting that they smoke in interview surveys due to the stigmatization of women smokers [25]. The women smoking prevalence appeared inconsistent among the three surveys, with the KNHANES results showing a higher percentage than the other two. This might be due to the different survey methods used. Unlike the other two surveys, which were conducted using a face-to-face interview method by surveyors who visited the household, the KNHANES data were collected in a moving vehicle where anonymity was guaranteed. In addition, any difference in the disclosure rates of smoking behaviors among women might have affected the results on socioeconomic inequalities in smoking prevalence and their time trends.

The KNHANES data sets also showed higher divergence than the KCHS and the SSSK data sets, possibly due to its insufficient sample size. This made it difficult to identify stable results after dividing the subjects into socioeconomic tertiles, even after combining biannual data and only analyzing the 
30-59 years age group. It was therefore impossible to evaluate the impacts of the recent anti-smoking policy using the KNHANES as a single source of data.

Gregoraci et al. [7] suggested that the magnitude of absolute inequalities in mortality due to smoking diminished among men, but increased among women. This finding could be interpreted as indicating that anti-smoking measures in the past have been more effective on men smokers [16]. Since women smokers in Korea tend to underreport their smoking status [25], it is unclear whether the results of women smoking prevalence and inequalities based on the collected information accurately reflect real-world circumstances. It may be helpful to consider the possibility that the increasing trend of inequalities in women may have been caused by disparities in the disclosure of smoking status across different socioeconomic groups. If, though, disclosure rates have not changed depending on socioeconomic status over the study period, then the results from women might be useful for evaluating the impacts of tobacco control policies. However, it is possible that disclosure rates have changed inconsistently across different socioeconomic groups. For instance, the increased socioeconomic status of women may have led them to be more open about their smoking status. Therefore, time trends in women smoking prevalence and its socioeconomic patterns may need to be assessed using urinary cotinine levels, which are a more objective indicator of an individual's smoking status.

Previous studies have repeatedly suggested that making cigarettes more expensive is the best way to reduce inequalities in smoking $[11,13,14,26]$. The Korean government made major moves to impose higher cigarette prices in 2004 and 2015. This policy did slow the growing pace of inequalities in smoking, although it failed to curb the absolute magnitude of inequalities [17]. The 2015 move was the sharpest hike in both relative and absolute terms, as the price of a pack of cigarettes increased from KRW 2500 (US\$ 2.3) to KRW 4500 (US\$ 4.0). This study showed that a slim reduction in inequalities was observed since the 2015 measure among men aged 30-59, the group with the largest population of smokers. However, relative inequalities among men, as well as both relative and absolute inequalities among women, did not seem to decrease according to the recent tobacco control policies. Relative inequalities in smoking have not seen any notable improvements despite the major effort by the government in 2015. A more aggressive tax policy for tobacco products seems necessary to further reduce socioeconomic inequalities in smoking.

\section{ACKNOWLEDGEMENTS}

This study was supported by the Mid-career Researcher Program of the National Research Foundation (NRF) funded by the Ministry of Science \& ICT (2017R1A2A2A05069746). This study was also supported by the Health Promotion Fund, Ministry of Health \& Welfare, Republic of Korea.

\section{CONFLICT OF INTEREST}

The authors have no conflicts of interest associated with the material presented in this paper.

\section{SUPPLEMENTAL MATERIALS}

Supplementary Materials 1-6: Tables S1-S6 are available at https://www.jpmph.org/.

\section{ORCID}

Youngs Chang https://orcid.org/0000-0003-4722-6894

Sanghyun Cho https://orcid.org/0000-0002-2081-1076

Ikhan Kim https://orcid.org/0000-0002-6428-4159

Jinwook Bahk https://orcid.org/0000-0002-7715-9955

Young-Ho Khang https://orcid.org/0000-0002-9585-8266

\section{REFERENCES}

1. GBD 2015 Tobacco Collaborators. Smoking prevalence and attributable disease burden in 195 countries and territories, 1990-2015: a systematic analysis from the Global Burden of Disease Study 2015. Lancet 2017;389(10082):1885-1906.

2. US Department of Health and Human Services. The health consequences of smoking - 50 years of progress: a report of the Surgeon General; 2014 [cited 2018 Nov 2]. Available from: https://www.surgeongeneral.gov/library/reports/50-yearsof-progress/full-report.pdf.

3. GBD 2013 Risk Factors Collaborators, Forouzanfar MH, Alexander L, Anderson HR, Bachman VF, Biryukov S, et al. Global, regional, and national comparative risk assessment of 79 behavioural, environmental and occupational, and metabolic risks or clusters of risks in 188 countries, 1990-2013: a systematic analysis for the Global Burden of Disease Study 2013. Lancet 2015;386(10010):2287-2323.

4. GBD 2016 Risk Factors Collaborators. Global, regional, and na- 
tional comparative risk assessment of 84 behavioural, environmental and occupational, and metabolic risks or clusters of risks, 1990-2016: a systematic analysis for the Global Burden of Disease Study 2016. Lancet 2017;390(10100):13451422.

5. Bosdriesz JR, Willemsen MC, Stronks K, Kunst AE. Socioeconomic inequalities in smoking cessation in 11 European countries from 1987 to 2012. J Epidemiol Community Health 2015; 69(9):886-892.

6. Mackenbach JP, Stirbu I, Roskam AJ, Schaap MM, Menvielle G, Leinsalu $\mathrm{M}$, et al. Socioeconomic inequalities in health in 22 European countries. N Engl J Med 2008;358(23):2468-2481.

7. Gregoraci G, van Lenthe FJ, Artnik B, Bopp M, Deboosere $P$, Kovács $\mathrm{K}$, et al. Contribution of smoking to socioeconomic inequalities in mortality: a study of 14 European countries, 1990-2004. Tob Control 2017;26(3):260-268.

8. Kivimäki M, Shipley MJ, Ferrie JE, Singh-Manoux A, Batty GD, Chandola $\mathrm{T}$, et al. Best-practice interventions to reduce socioeconomic inequalities of coronary heart disease mortality in UK: a prospective occupational cohort study. Lancet 2008; 372(9650):1648-1654.

9. Fawcett J, Blakely T. Cancer is overtaking cardiovascular disease as the main driver of socioeconomic inequalities in mortality: New Zealand (1981-99). J Epidemiol Community Health 2007;61(1):59-66.

10. Khang YH, Lynch JW, Jung-Choi K, Cho HJ. Explaining agespecific inequalities in mortality from all causes, cardiovascular disease and ischaemic heart disease among South Korean male public servants: relative and absolute perspectives. Heart 2008;94(1):75-82.

11. Brown T, Platt $S$, Amos A. Equity impact of interventions and policies to reduce smoking in youth: systematic review. Tob Control 2014;23(e2):e98-e105.

12. Brown T, Platt S, Amos A. Equity impact of population-level interventions and policies to reduce smoking in adults: a systematic review. Drug Alcohol Depend 2014;138:7-16.

13. Hill S, Amos A, Clifford D, Platt S. Impact of tobacco control interventions on socioeconomic inequalities in smoking: review of the evidence. Tob Control 2014;23(e2):e89-e97.

14. Pförtner TK, Hublet A, Schnohr CW, Rathmann K, Moor I, de Looze $M$, et al. Socioeconomic inequalities in the impact of tobacco control policies on adolescent smoking. A multilevel study in 29 European countries. Addict Behav 2016;53:58-66.

15. Cho HJ, Song YM, Smith GD, Ebrahim S. Trends in socio-economic differentials in cigarette smoking behaviour between 1990 and 1998: a large prospective study in Korean men. Public Health 2004;118(8):553-558.

16. Khang YH, Cho HJ. Socioeconomic inequality in cigarette smoking: trends by gender, age, and socioeconomic position in South Korea, 1989-2003. Prev Med 2006;42(6):415-422.

17. Khang YH, Yun SC, Cho HJ, Jung-Choi K. The impact of governmental antismoking policy on socioeconomic disparities in cigarette smoking in South Korea. Nicotine Tob Res 2009;11(3): 262-269.

18. Kim Y, Kang K, Lee K, Hwang S. Increase in cigarette taxation and use of national health promotion fund. Gwacheon: Ministry of Health and Welfare; 2003, p. 90-120 (Korean).

19. Cho KS. FCTC and tobacco control policy in Korea. Health Welf Policy Forum 2006;116:7-23 (Korean).

20. Chang Y. Trends in socioeconomic inequality in cigarette smoking prevalence according to recent anti-smoking policies in South Korea: use of three national surveys [dissertation]. Seoul: Seoul National University; 2018 (Korean).

21. Mackenbach JP, Kunst AE. Measuring the magnitude of socioeconomic inequalities in health: an overview of available measures illustrated with two examples from Europe. Soc Sci Med 1997;44(6):757-771.

22. Harper S, Lynch J. Measuring health inequalities. In: Oakes JM, Kaufman JS, editors. Methods in social epidemiology. 2nd ed. San Francisco: Jossey-Bass; 2006, p. 134-168.

23. Harper S, King NB, Meersman SC, Reichman ME, Breen N, Lynch J. Implicit value judgments in the measurement of health inequalities. Milbank Q 2010;88(1):4-29.

24. Organization for Economic Cooperation and Development. GDP per capita and productivity levels; 2017 [cited 2018 Nov 2]. Available from: https://doi.org/10.1787/36c96b83-en.

25. Jung-Choi KH, Khang YH, Cho HJ. Hidden female smokers in Asia: a comparison of self-reported with cotinine-verified smoking prevalence rates in representative national data from an Asian population. Tob Control 2012;21(6):536-542.

26. Brown J, West R, Beard E, Michie S, Shahab L, McNeill A. Prevalence and characteristics of e-cigarette users in Great Britain: findings from a general population survey of smokers. Addict Behav 2014;39(6):1120-1125. 